\title{
Sternal resection and reconstruction with a multi-functional modular prosthesis
}

\author{
Haifeng Wang, Chang Chen, Zhe Shi, Zhiyi Liu, Gening Jiang \\ Department of Thoracic Surgery, Shanghai Pulmonary Hospital, Tongji University, School of Medicine, Shanghai 200433, China \\ Correspondence to: Prof. Gening Jiang. Department of Thoracic Surgery, Shanghai Pulmonary Hospital. 507 Zhengmin Road, Shanghai 200433, \\ China. Email: jgnwp@aliyun.com.
}

\begin{abstract}
Reconstruction for a large area of sternal defect is challenging. The available sternal reconstruction techniques and materials have their limitations. We designed a modular sternal reconstruction system made of titanium alloy. This system was applied to a patient who received partial sternal resection for well-differentiated chondrosarcoma of the sternum. The sternal neoplasm measured $80 \times 70 \mathrm{~mm}^{2}$. After resection of most of the sternal body and adjacent ribs, standard modules of adequate types and sizes were chosen and assembled during operation. The operation lasted for $240 \mathrm{~min}$, with an intraoperative blood loss of $100 \mathrm{~mL}$. The patient was followed up for 32 months, while the prosthesis remained stable, with no signs of infection or looseness.
\end{abstract}

Keywords: Sternum; reconstruction; prosthesis; chondrosarcoma

Received: 02 November 2019; Accepted: 05 November 2019; Published: 06 November 2019.

doi: $10.21037 /$ ccts.2019.11.01

View this article at: http://dx.doi.org/10.21037/ccts.2019.11.01

\section{Introduction}

The sternum is the pivotal part of the chest wall, connecting clavicles and ribs on both sides. When the defect exceeds $5 \times 5 \mathrm{~cm}^{2}$ after sternal resection, reconstruction is justified to restore the bony chest wall. Titanium plates or meshes, Sandwich patches, auto- or allografts of bone materials, and $3 \mathrm{D}$ printing prostheses are often used for reconstruction. At present, there is no such ideal prosthesis that meets the requirement for sternal reconstruction, such as compatibility, mechanical strength, and tailorability. We designed a multi-functional modular system for chest wall reconstruction involving the sternum. By selecting and integrating a combination of pre-manufactured standard modules of different shapes and sizes, the anterior chest wall anatomy can be restored without the need for preoperative individualization design. This sternal reconstruction system was applied in one case of sternal neoplasms with satisfactory results.

\section{Case presentation}

\section{Design of modules}

The reconstruction system consisted of modules of manubrium, sternal bodies, ribs, and clavicles. Each module had 3 to 6 standard sizes. The sternal modules were connected in a tenon-mortise fashion, and the rib modules were connected to the sternal ones by a toothed gear structure, and screws fastened both. The rib modules could be placed in different angles by adjusting the toothed gear. All modules were made of titanium alloy, with rigidity no less than 260HV10. Shandong Weigao Orthopedic Materials Co. manufactured the products (Figures 1,2; Video 1).

\section{Clinical application}

A 48-year-old female patient was admitted due to "paroxysmal anterior chest pain for 20 years". A chest 


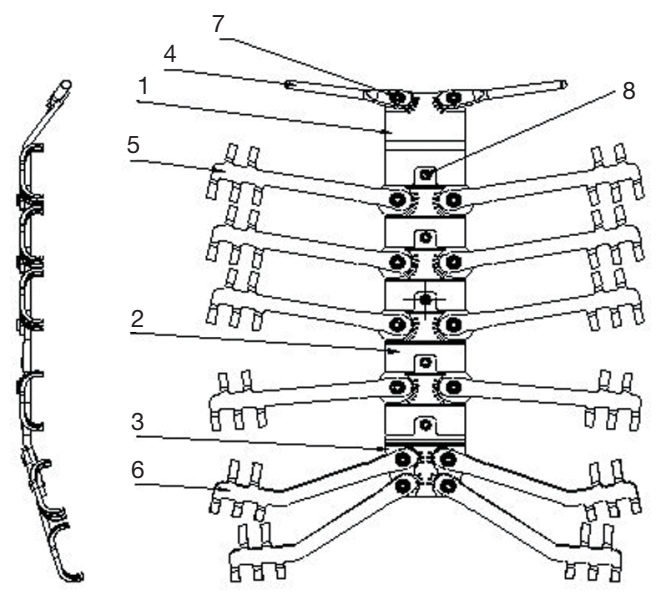

Figure 1 Overview of the design of the multi-functional modular sternal reconstruction prosthesis-reconstruction prosthesis for total sternal resection. 1, manubrium module; 2, sternal body module A; 3, sternal body module B; 4, intra-medullary needle for the clavicle; 5, costal claw (straight); 6, costal claw (curved); 7, connecting bolt/screw; 8 , fastening screw.

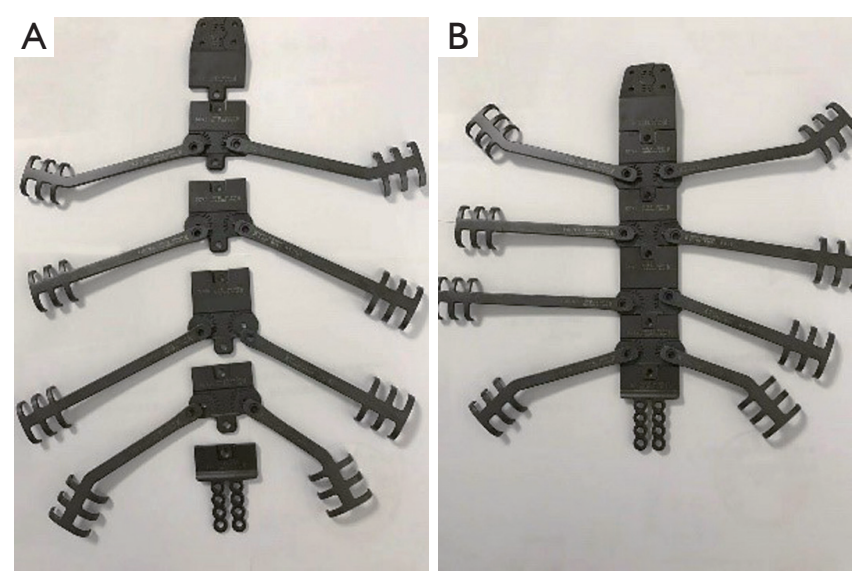

Figure 2 Sample of the multi-functional modular sternal reconstruction prosthesis. (A) Sternal reconstruction prosthesis dissolved; (B) sternal reconstruction prosthesis assembled.

CT scan revealed a mass measuring $77 \times 62 \mathrm{~mm}^{2}$ on the middle and lower sternal body, with focal calcification and lobulation. Sternal resection and reconstruction were performed after the exclusion of remote metastasis.

The operation lasted for $240 \mathrm{~min}$, with an intraoperative blood loss of $100 \mathrm{~mL}$. The semi-spherical neoplasm was in the middle and lower sternal body, measuring $80 \times 70 \mathrm{~mm}^{2}$, with an intact capsule, without macroscopic invasion to the pericardium, pleura, or other adjacent structures. The middle and lower sternal body was resected from the level of the superior border of the third ribs to just above the xiphoid process. The adjacent cartilaginous and osseous ribs of up to $5 \mathrm{~cm}$ in length were resected from the third ribs and below. The chest wall defect was reconstructed with the modular reconstruction system. Five sternum modules and four rib modules were used. The uppermost sternum module was connected to the remnant sternum, and the most inferior sternum module was connected to the remnant xiphoid. The rib modules were connected to the remaining rib stumps after being adjusted to the most desirable angle to the sternum modules so that the tip of each rib module was in line with the rib stump that it was supposed to be connected with. The connection of the prosthesis to the chest wall was firm and stable. The prosthesis was then covered with bilateral pedicled pectoralis major muscle flaps. Drainages were put in both chest cavities, and spaces above and beneath the muscle flaps. The pathological diagnosis was well-differentiated chondrosarcoma. The patient was discharged seven days after surgery. The patient had been followed-up for 32 months. No adjuvant therapy was given. The wound healed satisfactorily, without local infection, fluid accumulation or loosening of the prosthesis. There were no signs of recurrence or distant metastasis (Figure 3).

\section{Discussion}

Since the sternum is the pivotal part of the anterior chest wall, its reconstruction is difficult. Ideal sternal reconstruction requires restoration of the anatomical structure and function. It demands stability, relative impermeability, durability, chemical inertia, and compatibility (1-4). In the case of a tumor, the extent of resection needs to be determined during operation, usually exceeding the preoperative plan, making the reconstruction more difficult. Although there are a variety of materials and methods for sternal construction, none of them perfectly meets all the requirements.

Autologous bone transplantation is restricted by donor bone acquirement and surgical trauma (5-7). Allogenic bone transplantation needs to deal with risks of allograft infection, necrosis, and absorption, aside from requirements in preservation $(5,8-10)$. The traditional titanium plates cannot be overlapped to offer mechanical support on two dimensions $(11,12)$. The use of the Sandwich patch on large defects has the risks of dead space formation, effusion accumulation and even rupture of the prosthesis (13-15). Biosynthetic patches usually do not have enough strength 

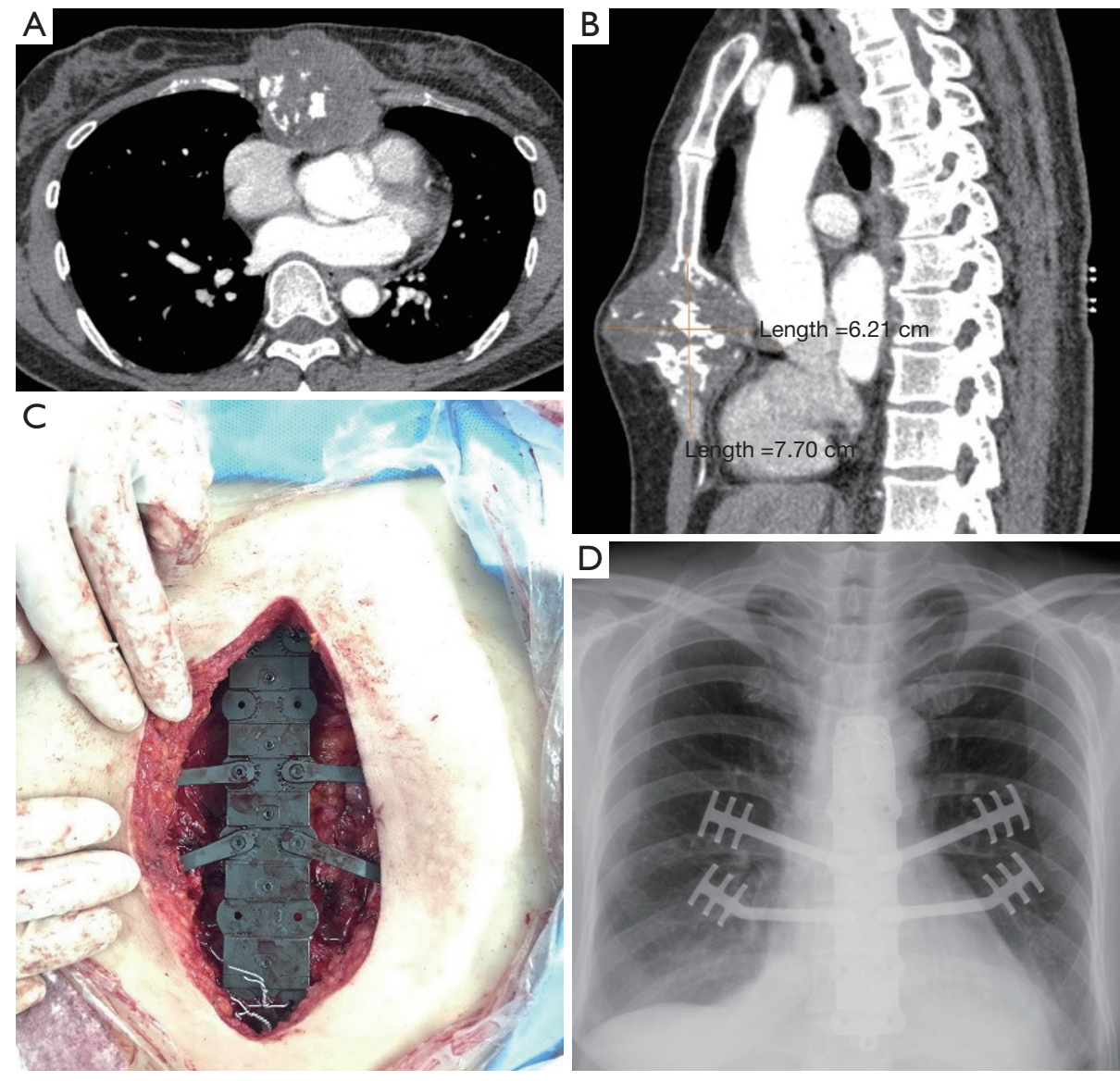

Figure 3 Case demonstration of sternal reconstruction. (A) Transverse view of the chest CT scan at the level of the sternal tumor; (B) sagittal view of the chest CT scan; (C) implantation of the sternal reconstruction prosthesis; (D) plain chest X-ray after surgery.

for the reconstruction of the large defect (16-18).

To overcome the shortcomings of the conventional ways of sternal reconstruction, we designed a novel system of the multi-functional modular prosthesis. Our reconstruction system is based on the notion of modularization. Standard modules of distinct parts of sternum, ribs, and clavicles are pre-manufactured in certain models and sizes. The reconstruction is performed by selecting and connecting modules of different models and sizes according to the defect after resection.

The modules are made of titanium alloy, whose mechanical strength is higher than human bone tissues so that the modules need not be exactly the size of the human sternum to supply enough strength. Therefore, all the modules are only different in length, while the width is unanimously $30 \mathrm{~mm}$, and the thickness $3 \mathrm{~mm}$. This design makes the manufacture and choice of the modules much easier, so are the connection between modules.
In this system, the sternum modules are connected by a tenon-and-mortise fashion and fastened with screws. The rib modules are connected to the sternum modules with a gear structure. Each rib module can be placed at the desired angle, fit into and restricted by the tiny notches on the sternum module, and then fastened by screw. There is no need to replace each resected rib with a rib module or keep the rib module at the same angle as the resected rib, if the reconstructed chest wall is stable.

Furthermore, there is no need to precisely predict the extent of resection for the design of the prosthesis preoperatively. One basic principle of oncological surgery is that the extent of resection shall be determined by exploration and frozen section. Unlike pre-manufactured customized prosthesis, this reconstruction system is assembled with standard modules selected on the operation table, making it easier to abide by the principles of oncological surgery. Since the modules are of standardized 
sizes, the preoperative waiting time can be minimized.

\section{Conclusions}

Our modular sternal reconstruction system is easy to manufacture, with no need for preoperative individualization. The prosthesis can be adjusted on the operation table to achieve a tumor-free margin. The connections between modules are easy and robust. By carefully selecting the proper modules, the anatomy and function of the anterior bony chest wall can be restored.

\section{Acknowledgments}

Funding: None.

\section{Footnote}

Conflicts of Interest: GJ serves as an unpaid Editor-in-Chief of Current Challenges in Thoracic Surgery (CCTS). CC serves as an unpaid Associate Editor-in-Chief of CCTS. HW serves as an unpaid Managing Editor of CCTS. The authors have no other conflicts of interest to declare.

Etbical Statement: The authors are accountable for all aspects of the work in ensuring that questions related to the accuracy or integrity of any part of the work are appropriately investigated and resolved. Written informed consent was obtained from the patient for publication of this manuscript and any accompanying images.

Open Access Statement: This is an Open Access article distributed in accordance with the Creative Commons Attribution-NonCommercial-NoDerivs 4.0 International License (CC BY-NC-ND 4.0), which permits the noncommercial replication and distribution of the article with the strict proviso that no changes or edits are made and the original work is properly cited (including links to both the formal publication through the relevant DOI and the license). See: https://creativecommons.org/licenses/by-nc-nd/4.0/.

\section{References}

1. Rocco G. Anterior chest wall resection and reconstruction. Operat Techn Thorac Cardiovasc Surg 2013;18:32-41.

2. Chang RR. Thoracic reconstruction. In: Thorne $\mathrm{CH}$, Beasley RW, Aston SJ, et al. editors. Grabb and Smith's Plastic Surgery. Philadelphia, PA: Lippincott Williams \&
Wilkins, 2007:665-9.

3. Lequaglie C, Massone PB, Giudice G, et al. Gold standard for sternectomies and plastic reconstructions after resections for primary or secondary sternal neoplasms. Ann Surg Oncol 2002;9:472-9.

4. Rocco G. Overview on current and future materials for chest wall reconstruction. Thorac Surg Clin 2010;20:559-62.

5. Zhang G, Liang C, Shen G, et al. Autogenous rib grafts for reconstruction of the manubrium after resection: technical refinements and outcomes. J Thorac Cardiovasc Surg 2014;148:2667-72.

6. Li W, Zhang G, Ye C, et al. Autogenous rib graft for reconstruction of sternal defects. J Thorac Dis 2014;6:1851-2.

7. Ren P, Zhang J, Zhang X. Resection of primary sternal osteosarcoma and reconstruction with homologous iliac bone: case report. J Formos Med Assoc 2010;109:309-14.

8. Dell'amore A, Nizar A, Dolci G, et al. Sternal resection and reconstruction for local recurrence of breast cancer using the sternal allograft transplantation technique. Heart Lung Circ 2013;22:234-8.

9. Nosotti M, Rosso L, Mendogni P, et al. Sternal reconstruction for unusual chondrosarcoma: innovative technique. J Cardiothorac Surg 2012;7:40.

10. Dell'Amore A, Cassanelli N, Dolci G, et al. An alternative technique for anterior chest wall reconstruction: the sternal allograft transplantation. Interact Cardiovasc Thorac Surg 2012;15:944-7.

11. Chong BK, Kim JB, Yun TJ. Simultaneous sternal reconstruction and tricuspid valve replacement in a patient with chronic osteomyelitis and absence of tricuspid valve. J Thorac Cardiovasc Surg 2012;144:e16-8.

12. Hajjar WM, Alnassar S, Abu-Daff SN, et al. A novel technique for the reconstruction of resected sternoclavicular joints: A case report with a review of the literature. Ann Thorac Med 2013;8:60-3.

13. Soysal O, Walsh GL, Nesbitt JC, et al. Resection of sternal tumors: extent, reconstruction, and survival. Ann Thorac Surg 1995;60:1353-8; discussion 1358-9.

14. Weyant MJ, Bains MS, Venkatraman E, et al. Results of chest wall resection and reconstruction with and without rigid prosthesis. Ann Thorac Surg 2006;81:279-85.

15. Tulner SA, van den Tol MP2, Meijer S. Stable construction of the sternum after broad radical resection of malignant tumours. J Surg Case Rep 2013. doi: 10.1093/jscr/rjt049.

16. Uflacker AB, Janis JE. The use of acellular dermal matrix in the correction of visible parasternal deformities after breast reconstruction. Plast Reconstr Surg 2010;126:34e-36e. 
17. Schmidt J, Redwan B, Koesek V, et al. Thoracic Wall Reconstruction with Acellular Porcine Dermal Collagen Matrix. Thorac Cardiovasc Surg 2016;64:245-51.

18. Kim CW, Cho HM, Son BS, et al. Neo-sternum

doi: $10.21037 /$ ccts.2019.11.01

Cite this article as: Wang H, Chen C, Shi Z, Liu Z, Jiang G. Sternal resection and reconstruction with a multi-functional modular prosthesis. Curr Chill Thorac Surg 2019;1:16. reconstruction using costal cartilage approximation and small Permacol patch repair in the treatment of Cantrell pentalogy: a case report. J Cardiothorac Surg 2015;10:40. 